\title{
Quadrupole Secondary Ion Mass Spectrometer for Simultaneous Detection of Positive and Negative Ions $\dagger$
}

\author{
S. Daolio ${ }^{1}$, B. Facchin ${ }^{1}$, C. Pagura ${ }^{1}$, A. Tolstogouzov ${ }^{1}$ and N. Konenkov ${ }^{2}$ \\ ${ }^{1}$ IPELP-CNR, Corso Stati Uniti 4, I-35127 Padova, Italy \\ ${ }^{2}$ Ryazan State Pedagogical University, Svoboda str. 46, R-390000 Ryazan, Russian Federation
}

\begin{abstract}
The purpose of this study was to design a quadrupole secondary ion mass spectrometer for simultaneous detection of both positive and negative ions of different mass. An original setup based on a quadrupole electrostatic deflector configuration that allows energy and spatial separation of all types of secondary ions was proposed. The device was modeled using the SIMION 3D program before building the prototype. Trajectory and acceptance calculations were provided. Positive and negative secondary ion mass spectra for molybdenum test samples were obtained. Copyright (C) 1999 John Wiley \& Sons Ltd.
\end{abstract}

Received 16 February 1999; Revised 1 March 1999; Accepted 2 March 1999

Elemental compositions of samples with surfaces that are quickly and irreversibly destroyed by primary ion beam bombardment and containing both electropositive and electronegative components may be better evaluated by simultaneous analysis of positive and negative secondary ions. This allows a direct correlation between signal intensities from all ion species sputtered at the same time from the same bombarded area.

Another interesting application of this technique is in the field of secondary ion mass spectrometry (SIMS) imaging, where simultaneous detection of secondary positive ions and secondary electrons may add topographical contrast to surface chemical images produced by ion bombardment.

A design for an instrument for fast atom bombardment SIMS provided with simultaneous detection of positive and negative secondary ions has already been described. ${ }^{1}$ In this spectrometer energy and space separation of the secondary ions with different polarity was obtained by a specially designed electrostatic analyzer at the output of a quadrupole mass filter consisting of four wire electrodes and a grounded shield case. The two diagonal wire electrodes have the same applied voltage and the others have an applied voltage of opposite polarity. The main drawbacks of such a spectrometer are the absence of simultaneous detection of positive and negative ions with different masses which is particularly important for depth profiling and imaging, and the energy separation of secondary ions after quadrupole that leads to a reduction in mass resolution.

Previously a quadrupole electrostatic deflector (QD) was successfully used as an energy analyzer in some installations, e.g. for research into photodissociation processes of molecular ions under ion and laser beams interaction, ${ }^{2}$ and in the collector system of a quadrupole secondary ion mass spectrometer. ${ }^{3,4}$

*Correspondence to: A. Tolstogouzov, IPELP-CNR, Corso Stati Uniti 4, 35127 Padova, Italy.

E-mail: alexander@pdadrl.pd.cnr.it

$\dagger$ Partially presented at the 1st European Workshop on Secondary Ion Mass Spectrometry, Munster, Germany, 4-6 October, 1998.
This paper reports the original design of a simple SIMS setup based on a particular QD configuration that allows simultaneous positive and negative secondary ion detection of different masses by two quadrupole analyzers.

\section{EXPERIMENTAL}

A schematic layout of the secondary ion mass spectrometer is shown in Fig. 1. The unit consists of a newly designed quadrupole electrostatic energy analyzer/deflector (1), an inert gas ion gun model 3M (Kratos Analytical Instruments, Manchester, England) (2), two quadrupole mass analyzers model QMA 421 (Balzers AG, Furstentum Liechtenstein) $(3,4)$ with additional constructed Einzel lenses $(5,6)$, secondary electron multipliers (SEM) $(7,8)$ as detectors for positive and negative secondary ions, and an analyzing sample (9). The output signals from the SEMs are measured by a pulse-counting system and processed by a PC with Balzers Quadstar 421 Software, which also controls the quadrupole electronics.

\section{RESULTS AND DISCUSSION}

The problems of using a two-dimensional quadrupole electrostatic field as an energy analyzer and achromatic deflector of charged particles have been considered in many articles. ${ }^{2-6}$ In accord with the potential $V(x, y)$ into the quadrupole deflector ${ }^{5}$ of cylindrical symmetry (Fig. 1), it is calculated from the following expression:

$$
V(x, y)=\beta V_{\mathrm{k}}\left(x^{2}-y^{2}\right) R^{-2}+V_{0}
$$

where ' $\beta$ ' is a factor depending on the shape of the pole electrodes, $\pm V_{\mathrm{k}}$ is the potential applied on opposite diagonal electrodes and $V_{0}$ is the constant potential outside the deflector which determines the kinetic energy of the ions flying in the QD. Hyperbolic electrodes can be replaced by segments of cylinders of radius $r=1.148 R$, with a sufficient degree of accuracy, where $R$ (quadrupole field radius) is the radius of the circle tangent to quadrupole electrodes. ${ }^{5}$ The 


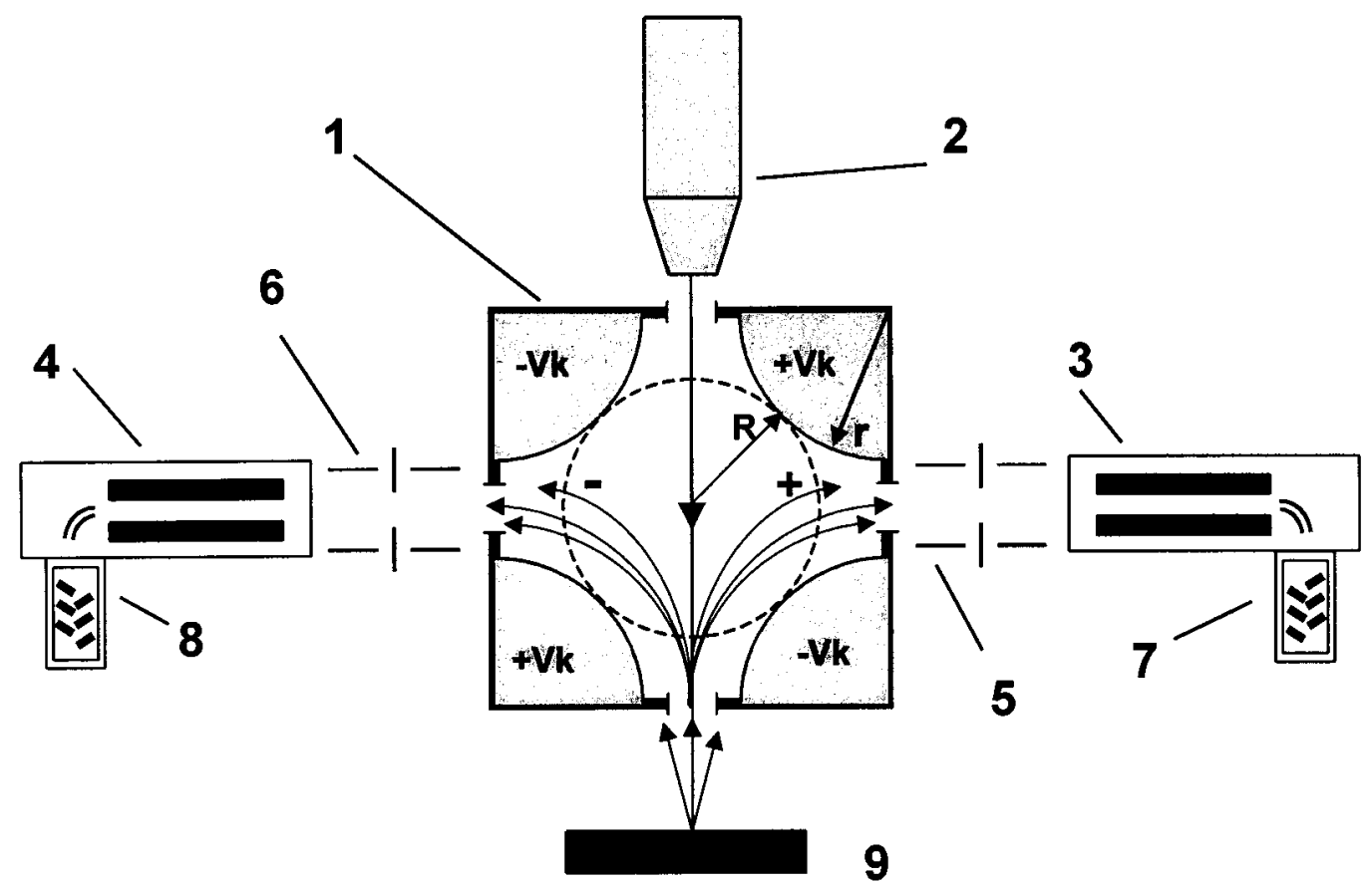

Figure 1. Secondary ion mass spectrometer with a quadrupole deflector for simultaneous detection of positive and negative secondary ions.

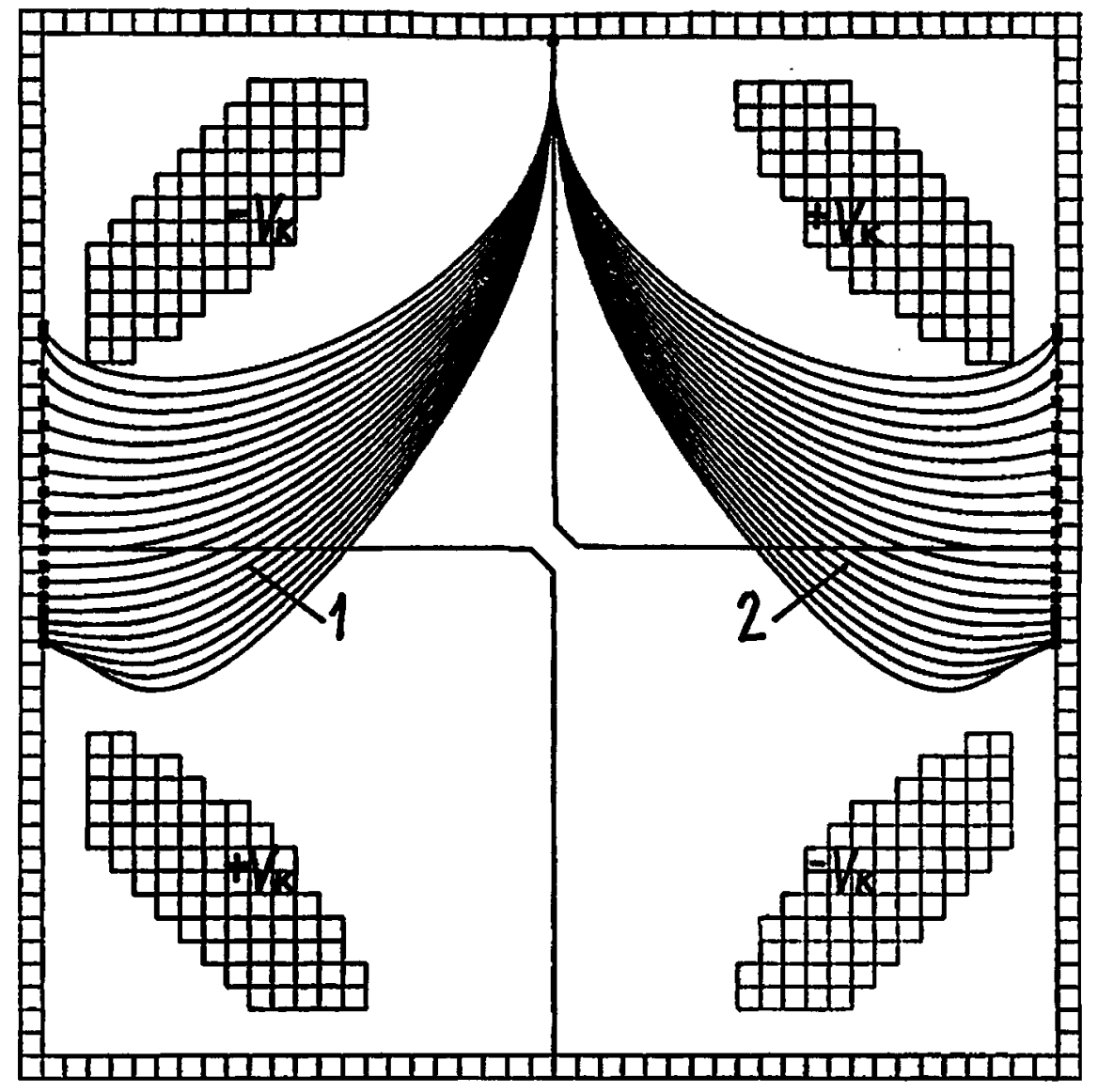

Figure 2. Trajectory evaluation of the positive (1) and negative (2) secondary ions into the quadrupole deflector: the secondary ion energy is $q V_{0}=2 \pm 1 \mathrm{eV}$ and deflector pole potentials are $V_{\mathrm{k}}= \pm 3.5 \mathrm{~V}$. Simulations were performed with the SIMION 3D program. 


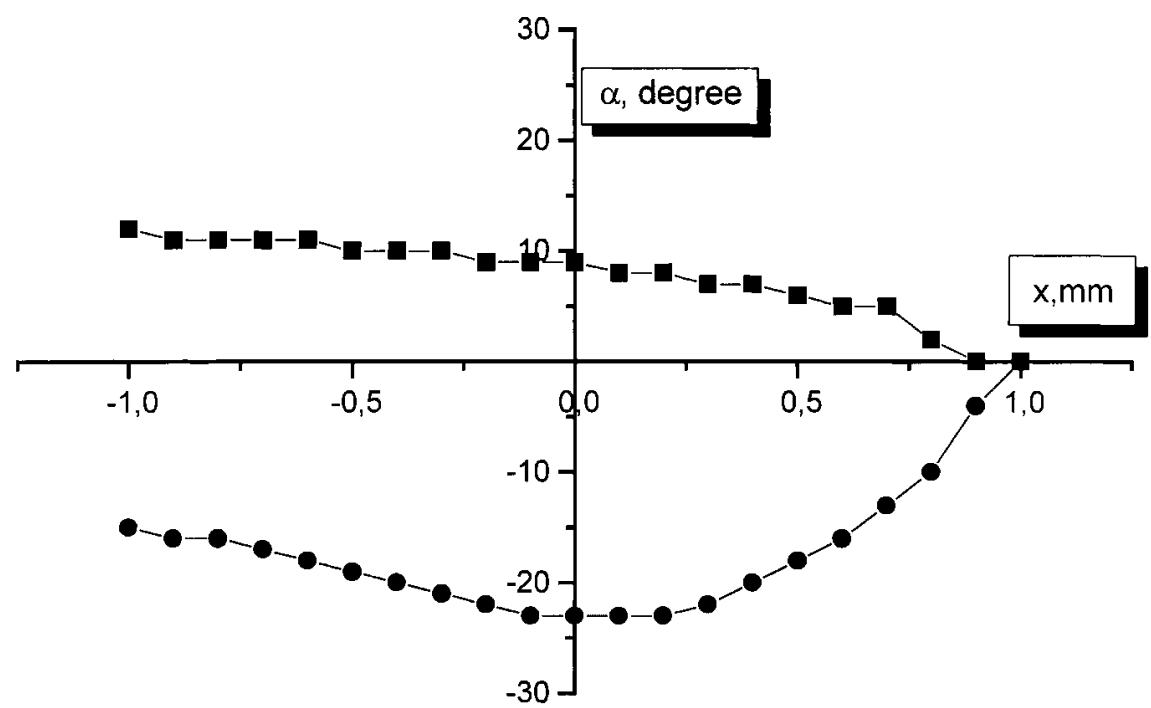

Figure 3. Result of QD acceptance calculations: the secondary ion energy $q V_{0}=10 \mathrm{eV}$; the deflector pole potentials $V_{\mathrm{k}}=1.74 V_{0}$; the diameter of the output diaphragm is $2 \mathrm{~mm}$; $\alpha$ is the angle between the secondary ion trajectories and a normal to the input diaphragm; $x$ is the distance between the secondary ion start point and the center of the input diaphragm. Simulations were performed with the SIMION 3D program.
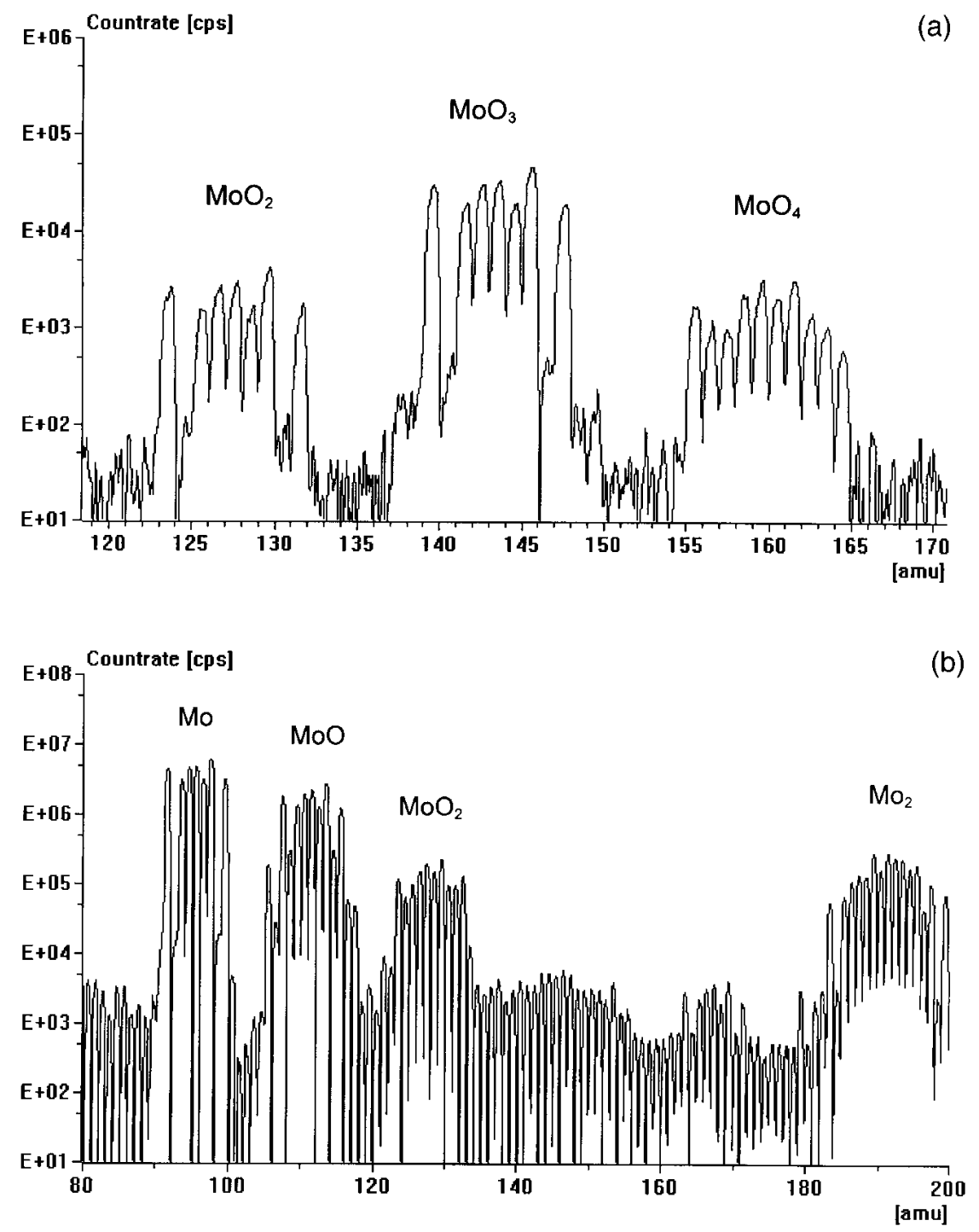

Figure 4. Secondary ion mass spectrum of a molybdenum test sample obtained using the QD system: (a) negative and (b) positive secondary ions. 
factor ' $\beta$ ' thus becomes equal to 1 . Theoretical calculations have shown ${ }^{2}$ that the maximum ion transmission through this deflector is provided at the relative quadrupole electrode potential $\left|V_{\mathrm{k}}\right|=k V_{0}$, where $k=1.245$ and $q V_{0}$ is the kinetic energy of the analyzed ions. By means of ion trajectory analysis, ${ }^{3}$ it can be demonstrated that the maximum diameter of the input QD diaphragm should not exceed $0.34 R$.

The experimental device was modeled using the SIMION $3 \mathrm{D}$ program ${ }^{7}$ before building the prototype. The quadrupole field radius $R$ was chosen equal to $9 \mathrm{~mm}$, and overall dimension of the QD was about $22 \mathrm{~mm}$. The diameter of all input and output diaphragms was $2 \mathrm{~mm}$. Figure 2 depicts some results of our ion trajectory calculations. It shows that the maximum transmission through the QD was realized with the coefficient ' $k$ ' $\simeq 1.7-1.8$. The influence of extended boundary electrical fields on a real QD system, not considered in theoretical calculation, ${ }^{2}$ was taken into account in this simulation. The energy resolution was about 15-20\% full-width at half-maximum (FWHM) for a common secondary ion energy $\left(\mathrm{e} V_{0}=1-50 \mathrm{eV}\right)$. Results of the QD acceptance calculations for optimum relative potential $V_{\mathrm{k}}$ are shown in Fig. 3. In the first approximation all secondary ions with trajectory between $\pm 10^{\circ}$ to the normal of the input diaphragm (sample surface) pass through the QD.

The QD filter was positioned between the final ion gun electrode and the sample, without any disturbance to the primary ion focus, allowing a very compact arrangement for the analytical chamber. Using two separated quadrupole mass filters for positive and negative secondary ion detection avoids SEM potential switching which is necessary when a single quadrupole is used. High voltage SEM potential switching causes instability in multiplier responses and shortens its lifetime. Besides, a SIMS setup with the proposed quadrupole deflector system provides a normal incidence angle for the primary ion beam on the sample surface. Under these experimental conditions it is possible to obtain a very high depth profiling resolution by SIMS for multilayer super thin structures and ultra shallow implanted samples. ${ }^{8}$

Some experimental results of an application of the QD setup described above for analyses of a molybdenum test sample are shown in Figs $4\left(\mathrm{a}\right.$ and b). $\mathrm{Ar}^{+}$ions $(4 \mathrm{keV}, 1 \mu \mathrm{A})$ were used as primary ions. An additional oxygen flux for chemical activation of both positive and negative secondary ion emission was also employed. The total vacuum in the analytical chamber with this flux was not less than $5 \times 10^{-4}$ $\mathrm{Pa}$. Experimental sensitivity for ${ }^{98} \mathrm{Mo}^{+}$secondary ions was approx. (5-8) $\times 10^{3} \mathrm{cps} / \mathrm{nA}$ and for negative mode $\left({ }^{98} \mathrm{Mo}^{16} \mathrm{O}_{3}\right)$ was not less than $50-100 \mathrm{cps} / \mathrm{nA}$. The experimental value of the QD coefficient $k \approx 1.75$ corresponds to our computer evaluation with good accuracy.

\section{CONCLUSIONS}

The proposed original design for a secondary ion mass spectrometer allows energy and spatial separation for simultaneous detection of both positive and negative secondary ions with different masses and is suitable for the arrangement of a simple and compact instrument for routine characterization of materials. This setup will be used in our laboratory for the quantitative elemental analysis by SIMS of precious metal alloys. After some improvements and modernization of the primary ion gun system, low energy depth profiling analysis with high resolution by SIMS will be the goal of this research program.

\section{REFERENCES}

1. H. Hayashi, T. Satake, K. Kaneko and K. Nagai, Appl. Surface Science 70/71, 287 (1993).

2. H. D. Zeman, Rev. Sci. Instrum. 48, 1079 (1977).

3. S. S. Volkov, N. V. Konenkov, G. A. Mogil'chenko, A. B. Tolstogouzov and G. I. Shagimuratov, Prib. Tekh. Eksp. (Rus.) No. 6, 140 (1989).

4. A. B. Tolstogouzov and E. V. Mamontov, Instrum. and Experiment. Techniq. 39, 723 (1996).

5. P. H. Dawson, Quadrupole Mass Spectrometry and Its Applications, Elsevier, (1976).

6. C. Schmidt, Rev. Sci. Instrum. 41, 117 (1970).

7. David A. Dahl, SIMION 3D Version 6.0 User's Manual, INEL-95/ 0403 (1995).

8. J. B. Clegg, N. S. Smith, M. G. Dowsett, M. J. J. Theunissen and W. B. de Boer, J. Vac. Sci. Technol. A 14, 2645 (1995). 